\title{
Design of FH/TH hybrid spread spectrum system based on FPGA
}

\author{
Longjun Liu \\ School of Information, yunnan University \\ Kunming, China \\ 893817028@qq.com \\ Qianlin Liu \\ School of Information, yunnan University \\ Kunming, China \\ liuqianlin@sina.com
}

\author{
Hongwei Ding \\ School of Information, yunnan University \\ Kunming, China \\ dhw1964@163.com \\ Weifeng Zhang \\ School of Information, yunnan University \\ Kunming, China \\ 767950080@qq.com
}

\begin{abstract}
According to the present situation of the application of frequency hopping system, the existence question and the characteristic of the frequency hopping,time hopping system, based on the thought of complementary advantages and disadvantages, this paper puts forward a hybrid spread spectrum. It is to combine two kinds of spread spectrum with frequency hopping and time hopping to constitute a FH/TH hybrid spread spectrum system. We have conducted simulation and FPGA implementation for the FH/TH hybrid spread spectrum system and verify the validity of the design system.
\end{abstract}

Keywords-FH/TH; hybrid spread spectrum; simulation; FPGA

\section{INTRODUCTION}

As two aspects of the jumping expansion system, frequency hopping and time hopping have their own advantages and disadvantages. This makes the two jump way

National Natural Science Foundation (61461053, 61461054, 61072079), Yunnan Province Natural Science Fund (2010CD023), Yunnan University Fund (NO. XT412004). on the development and application is not the same. The principle of frequency hopping is to make the carrier that is used to send the modulation jump on the different frequency, which makes the frequency of the emission signal jump continuously in a wide frequency range [1]. As a result, it is difficult to conduct frequency interference and intercept decoding for the frequency hopping signal in the case without knowing the frequency-hopping pattern. Frequency hopping system has many fine properties, such as strong anti-interference ability, high confidentiality, strong resistance to multipath fading and multiple access capability, etc. These properties make it rapid development and wide application in the field of military and mobile communication. However, due to the extremely wide range of applications of frequency hopping technology, it has formed equipment in different frequencies. According to relevant information, foreign armies has formed a valid tracking ability to low and medium speed (500/second) frequency hopping communications with frequencies under $500 \sim 1000 \mathrm{MHz}$ [3]. This will make the advantage of using frequency hopping system not be able to play. With the development of ultra wideband UWB 
communication technology, jump modulation gets attention and applications [4]. Jump system is not really the spectrum broadening. It controls the time jumps of sending a frame and has the characteristics of signal concealed. Combining the frequency hopping and time hopping together to constitute a FH/TH hybrid spread spectrum system, which can effectively overcome the concealment of the frequency hopping signal is poor and easy to be found. It also can enhance the capacity of the multiple access of the system, improve the system as a whole new and broaden the application of the system.

FPGA has logical resources, fast processing speed, field programmable and many other advantages. It can effectively solve the communication system development difficult, long development cycle, hardware circuit is not easy to modify and many other problems, which determines the advantage of using FPGA in system development.

\section{THE PRINCIPLE OF FH/TH HYBRID SPREAD SPECTRUM SYSTEM}

The FH/TH hybrid spread spectrum system increases the emission signal transmission time to jump control functions on frequency-hopping system. In the sending end, for the information to be sent $\mathrm{m}(\mathrm{t})$, jump frequency modulation firstly to spread spectrum signal, and then control RF switch jump sent by timing the jump time-code. At the receiving end, for the received spread spectrum signal $x(t)$, jump the spread spectrum demodulation to recovering the original information sequence order, and then get for low frequency data information by its frequency hopping spread spectrum. The principle of $\mathrm{FH} / \mathrm{TH}$ hybrid spread spectrum system is shown in figure 1.

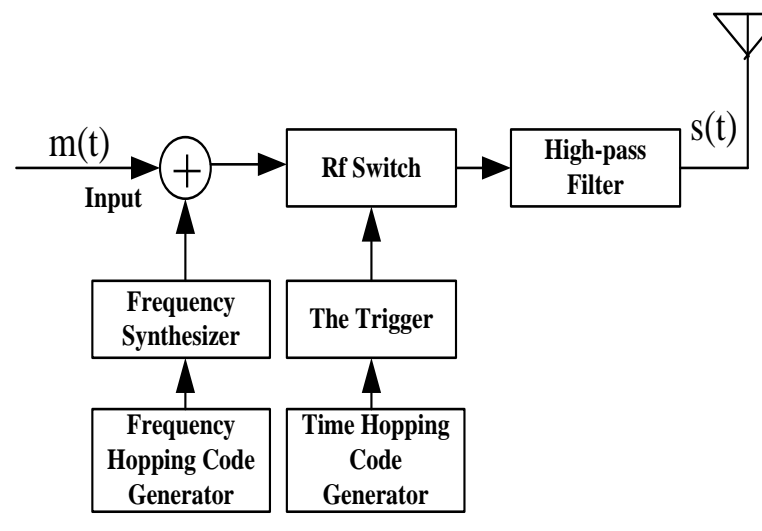

(a)The Sender

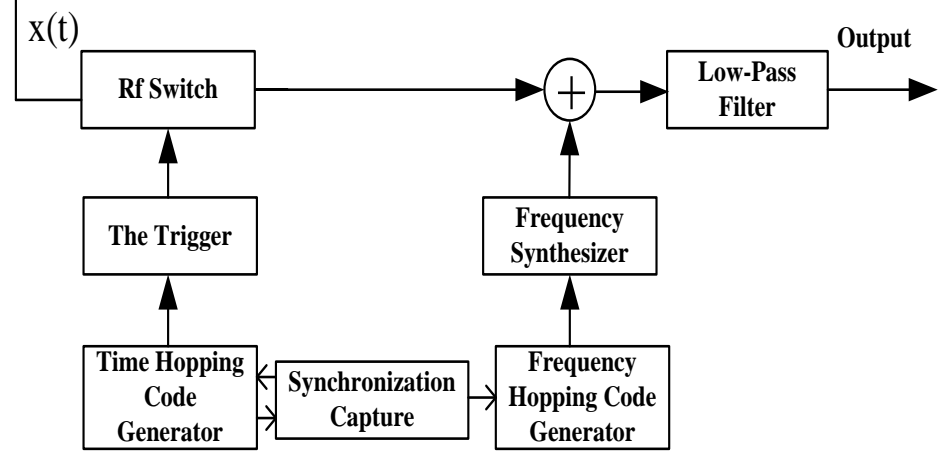

(b)The Receiving End

Fig.1 The principle diagram of FH/TH hybrid spread spectrum system

\section{THE FPGA DESIGN OF FH/TH HYBRID SPREAD SPECTRUM SYSTEM}

\subsection{SENDING SUBSYSTEM}

In the spread spectrum system, pnf module is frequency hopping code generator module that generates the FH code for different carrier frequency hopping modulation control; pnt module is time-hopping code generator module that generates code for spread spectrum signal timing control; fhmod module is frequency-hopping modulation module that realizes the frequency-hopping modulation; thmod module is jump modulation module that aims to realize the jump spread-spectrum signal modulation. Input port data is information input and output port mod_out is spread spectrum modulation output. 


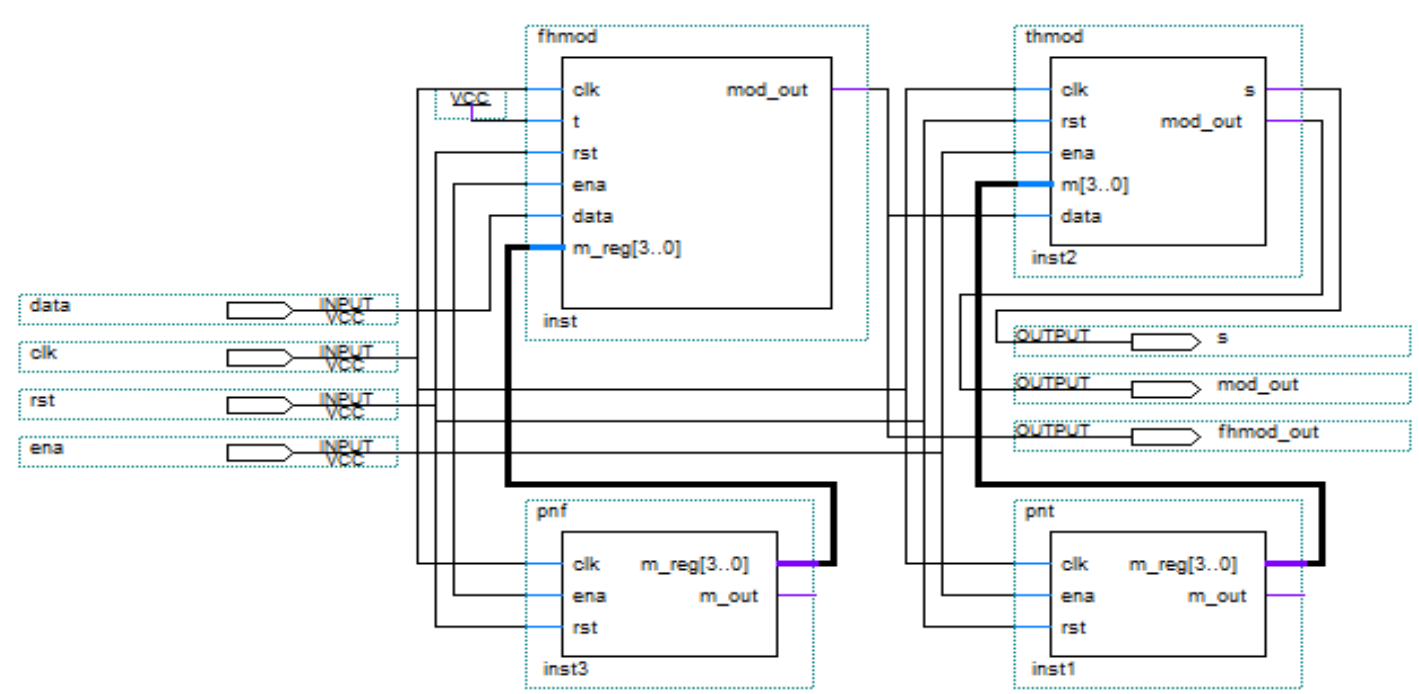

Fig.2. Spread spectrum send subsystem

\subsection{RECEIVER SUBSYSTEM}

In FH/TH hybrid spread spectrum system, modules of epnt, epnf module, syn, themod module and fhemod module constitute a received spread spectrum system. Among them, epnt module and epnf module are local jumps time codes and local frequency-hopping code generator module, generating the jump spread spectrum and frequency-hopping spread spectrum expansion codes. The syn module that uses sliding-window correlation detection method is synchronization of signal detection modules to achieve synchronous signal capturing [5]. themod module and fhemod module respectively are time-hopping spread spectrum module and frequency-hopping spread spectrum module, implementing a spread spectrum signal in the receiver jumps spread spectrum and frequency-hopping spread spectrum function. Emod_out output port is system expansion of output information.

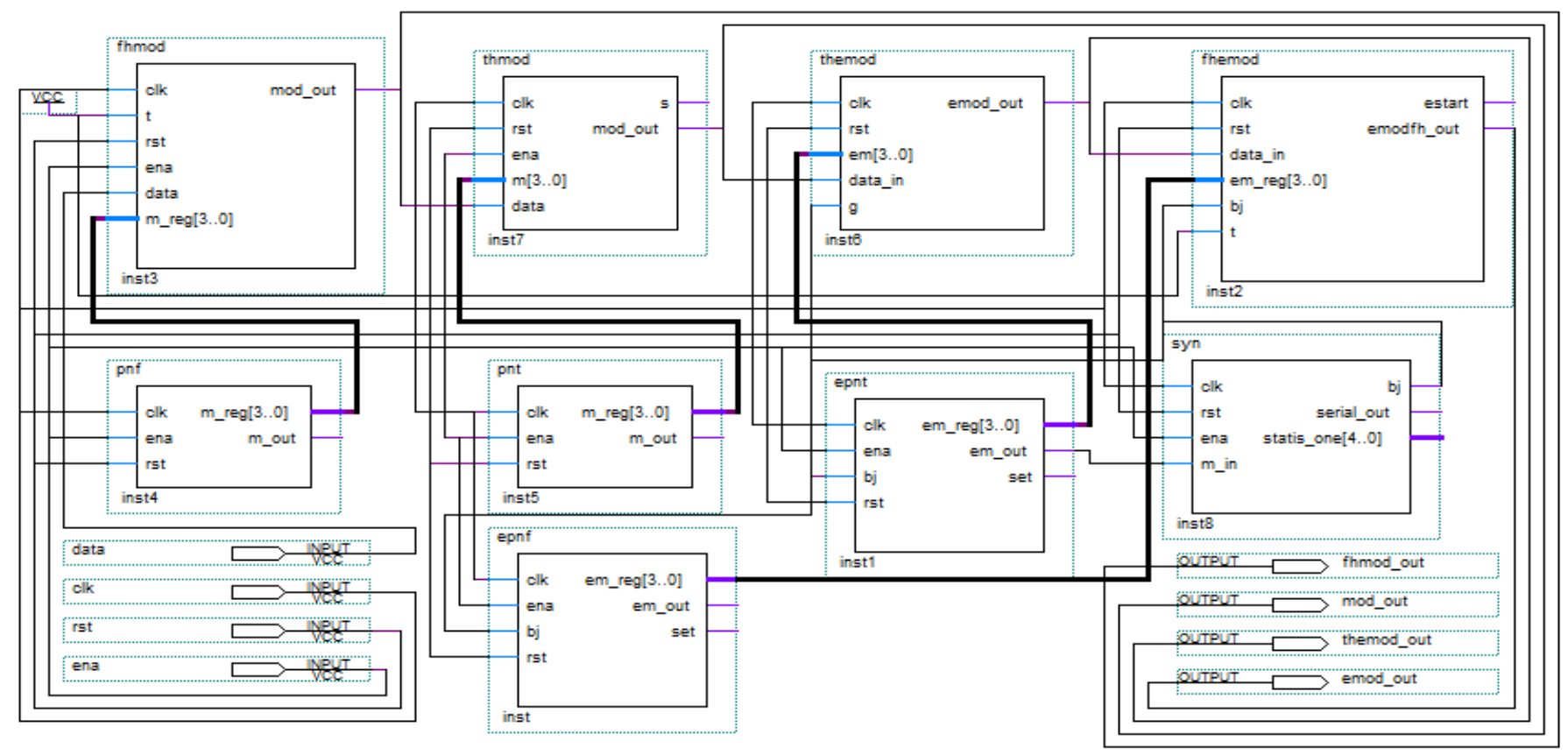

Fig.3 FH/TH hybrid spread-spectrum system 


\section{FH/TH HYBRID SPREAD SPECTRUM SYSTEM SIMULATION}

\section{AND ANALYSIS}

It is is advantaged for the further improvement and optimization of the system through a simulation of the design system, verify the validity and rationality of the design. As is shown in Fig.4 Send system simulation results, fhmod_out is the output frequency modulation and mod_out spread-spectrum output is sending subsystem, two of which realize the spreading of information and timing jumps. As is shown in Fig.5 FH/TH hybrid spread spectrum system simulation results, the mod_out is time-hopping spread spectrum output and the output signal waveform is the same to the frequency hopping modulation fhmod_out. Emod_out is $\mathrm{FH} / \mathrm{TH}$ hybrid spread-spectrum despreading output. Compared with the information data of the sender, its signal has some delay. The delay here mainly comes from the sequence circuit it's own transmission, handling latency and jump system to achieve the system time delay that the sequential jump brings [6]. In addition, emod_out initial stage has a small section of ber. This is because in the algorithm to the initial stage, the synchronization signal is not captured and the system doesn't achieve stable synchronization error when decoding. This will not affect synchronous information correctly spread. In addition to the above two points, the expansion of output signal and the sender input information system solution is completely consistent. Implement The designed system on FPGA and the oscilloscope display the result is the same with the simulation result. This means that the designed FH/TH hybrid spread spectrum system is correct, reasonable and feasible.

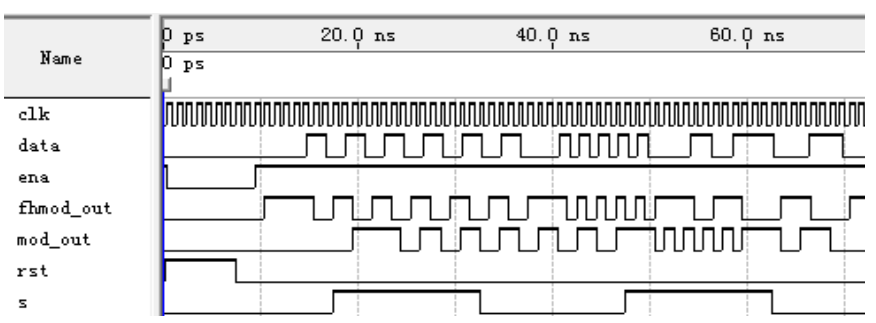

Fig.4 Send system simulation results

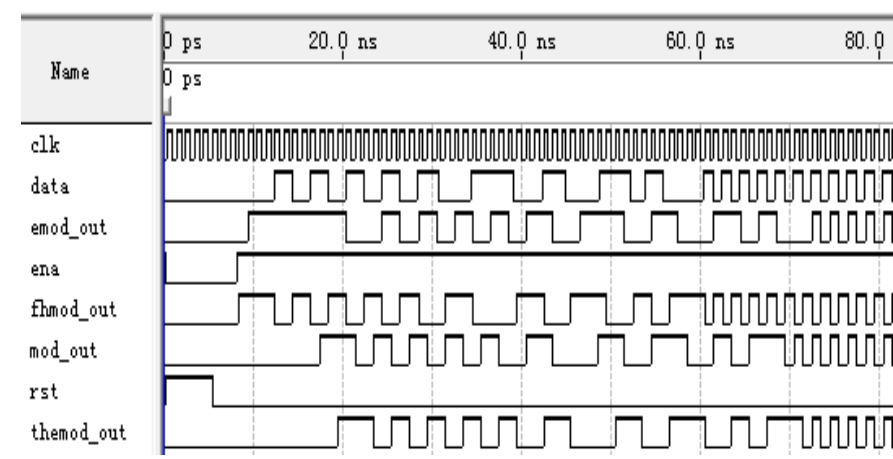

Fig.5 The system simulation results

\section{CONCLUSION}

According to the application of frequency hopping system status, problems as well as the characteristics of frequency hopping and time hopping system, this paper proposes to combine the two spread spectrum together to form the FH/TH hybrid spread-spectrum system. We do the system FPGA design by the combination of a hardware description language Verilog HDL and schematic diagram and simulation to verify the system is feasible.

\section{REFERENCES}

[1] Liu Jiangyong. Working principle and the method of spread spectrum communication [J]. Journal of information and communication, 2012 (5) : 189-190.

[2] Bai Mu, Zhou Jie. Spread spectrum communication principle, working mode, characteristics and application [J]. Power system communication, 2002 (4) : 36 to 39.

[3] Jiang Jianmin. Jump when the spread spectrum communication and its anti-jamming performance are analysed [J]. Modern military communications, 2002, 10 (1) : 1-4.

[4] Du Hongfeng, Liang Zhaohui, Zhou Zheng. PERFORMANCE ANALYSIS OF TIME-HOPPING M-ARY UWB WIRELESS SYSTEM USING V-BLAST ALGORITHM[J].JOURNAL OF ELECTRONICS(CHINA),2004,21(6):500-504.

[5] Liang Dongpo, Zhou Andong, Qu Xiaoxu, Wang Yongbin. Based on the FPGA implementation of shortwave fh synchronization when jumping [J]. Journal of signal and information processing, 2012, 42 (5) : 24-26.

[6]Abusayeed Saifullah,You Xu,Chenyang Lu,Yixin Chen.End-to-End Communication Delay Analysis in Industrial Wireless Networks[J].IEEE Transactions on Computers,2015(5):1361-1374. 